\title{
Fast Neutron and Gamma-Ray Detectors for the CSIRO Air Cargo Scanner
}

\author{
J.E. Eberhardt, A.J. McEwan, D. Milinkovic, V. Sharp, B.D. Sowerby* and J.R. \\ Tickner \\ CSIRO Minerals, Private Mail Bag 5, Menai NSW 2234 Australia \\ E-mail: brian.sowerby@csiro.au
}

The Commonwealth Science and Industrial Research Organisation (CSIRO) has been working with Australian Customs Service to develop a scanner capable of directly scanning airfreight containers in 1-2 minutes.

A critical aspect of the CSIRO Air Cargo Scanner has been the development of fast neutron and gamma ray detector arrays and electronics with the following desirable characteristics:

- High efficiency, particularly for fast neutrons as the brightness of conveniently available neutron sources is low;

- Small detector size. The size of the individual elements of the detector array determines the spatial resolution of the transmission images. A small detector element is desirable to provide sharper images but at the expense of increasing cost and complexity.

- The cost of detector and signal processing electronics should be less than about US\$200 per detector channel.

The CSIRO Air Cargo Scanner at Brisbane Airport contains 704 neutron detectors and 352 gamma detectors assembled on array boards. The fast neutron detectors in the scanner use orange-light emitting plastic scintillators $(20 \times 20 \times 75 \mathrm{~mm})$ and the gamma-ray detectors use CsI(Tl) scintillators $(10 \times 10 \times 50 \mathrm{~mm})$. Each detector uses photodiode readout and was coated to maximise light collection at the photodiode. A significant technical challenge in the current project was to develop low noise/high gain amplifiers at a relatively low cost.

International Workshop on Fast Neutron Detectors

University of Cape Town, South Africa

April 3-6, 2006 


\section{Introduction}

CSIRO has developed the fast-neutron/gamma-ray radiography (FNGR) method for the rapid scanning of air freight containers [1,2]. By combining $14 \mathrm{MeV}$ fast-neutron and ${ }^{60} \mathrm{Co}$ gamma-ray radiographic measurements, images are produced that represent both the density and composition of the contents of a container. CSIRO has installed a full-scale commercial prototype scanner at an Australian Customs Service purpose-built facility at Brisbane International Airport. Customs are undertaking a trial of the technology in a real-time working environment as well as a rigorous evaluation of the technology and associated business processes.

The key requirements of detectors for the CSIRO Air Cargo Scanner are:

- high efficiency for both $14 \mathrm{MeV}$ neutrons and ${ }^{60} \mathrm{Co}$ gamma rays (1.17 and $1.33 \mathrm{MeV}$ ).

- good spatial resolution, particularly for the gamma ray images as they are used to provide shape and density information [1]

- neutron detectors to be insensitive to both low energy scattered neutrons and gamma-ray background

- height of detector array to be greater than about $4300 \mathrm{~mm}$

- for systems employing, say, 1000 individual detectors, the cost per detector channel should be low and preferably less than about US\$200.

- good gain stability

- good high-count-rate performance with predictable deadtime

- reliability

A number of detection methods have been previously investigated for fast neutron radiography, including the use of scintillating screens with various readout schemes [3-6]. Unfortunately these detection methods do not meet all the requirements listed above, especially efficiency.

\section{Fast Neutron Detector Array}

High neutron detection efficiency is particularly important as the brightness of conveniently available neutron sources is low and scanning times are short (about 1-2 minutes per container). The most efficient fast neutron detectors are those based on proton recoil in plastic (or liquid) scintillators. Readout of the light from the plastic scintillators is performed using silicon photodiodes. Compared to photomultiplier tubes, silicon photodiodes are inexpensive, compact and rugged. In addition they only require low bias voltage ( $\sim 50$ volts) and they do not require gain stabilisation. The spectral response of silicon photodiodes is well matched to that of orange plastic scintillators that are available from a number of manufacturers.

Each plastic scintillator was $20 \times 20 \times 75 \mathrm{~mm}$ (Figure 1). The $75 \mathrm{~mm}$ length of the scintillator was chosen to provide a good compromise between neutron attenuation and light collection. 
The reflective coating of the scintillators was optimised to maximise light collection at the photodiode and to provide optical decoupling between neighbouring pixels. The conventional photodiodes we used are inexpensive but have no internal gain and produce a very small signal (a few thousand electrons) for fast neutrons. Signals from each detector channel are integrated using a custom-designed low-noise charge-sensitive preamplifier [7]. The millivolt signals from the preamplifiers are amplified and gaussian filtered to optimise the signal to noise ratio at about 10:1 to 20:1. Window discriminators on each channel select the unipolar signal pulses and reject noise from the preamplifier and from most of the radiation interactions directly in the silicon photodiodes. The discriminator upper and lower levels can be adjusted individually for each channel under computer control. There are no individual adjustments in the preamplifier/shaping amplifier channels.

Blocks of 16 neutron detectors are mounted onto a single circuit board and share common power supply and communication connections. The complete neutron detector array in the Brisbane scanner comprises 4 columns of 11 boards (Figure 2), for a total of 704 detector elements. Pulse height spectra from one block of 16 detectors irradiated with $14 \mathrm{MeV}$ neutrons are shown in Figure 3. The neutron count rates per detector above the lower level discriminator with no intervening cargo are typically about 800 counts per second.

\section{Gamma-Ray Detector Array}

The gamma-ray detector array uses $\mathrm{CsI}(\mathrm{Tl})$ scintillator elements measuring 10x10x50 mm with the long axis aimed at the gamma source. The peak scintillation wavelength of CsI(Tl), around $550 \mathrm{~nm}$, is well matched to the photodiode response. Reports in the literature on the optimisation of CsI(Tl) scintillator/ photodiode detector systems have shown that the coating of the scintillator in reflective layers gave optimum results $[8,9]$.

Due to their smaller size, 32 gamma-ray detectors are mounted on each circuit board, which has the same footprint as a 16 channel neutron detector board. The gamma-ray detector array in the Brisbane scanner has a single column containing 11 boards, for a total of 352 detector elements. The detector electronics are similar to the neutron detector electronics except that smaller photodiodes are used and bipolar shaping is employed. The gamma-ray count rates per detector above the lower level discriminator with no intervening cargo are typically about 23,000 counts per second.

\section{Detector Readout and Imaging}

As the detector system comprises narrow, vertical columns of detectors, the cargo is moved through the fan-beams of radiation by a chain conveyor to build up a 2-dimensional image line by line. Control and readout of the neutron and gamma-ray boards is managed via a custom-designed, high-speed serial interface. Each of the boards in a column is daisy-chained together and connected to a PC. The temperature of the detector arrays is controlled at around 
$20^{\circ} \mathrm{C}$. The accumulated counts from each detector channel are read out each time the cargo on the chain conveyor has traversed $5 \mathrm{~mm}$. The chain conveyor is typically operated at a speed of $1-$ $2 \mathrm{~m} / \mathrm{min}$ to achieve scanning times of approximately 1-2 minutes per ULD.

\section{Summary}

CSIRO has developed custom-designed neutron and gamma-ray detector systems, each comprising a large number of small detector elements. The size of the individual detector elements determines the final resolution of the scanned images. Both systems use scintillators to convert the incident radiation into a light pulse, which is then read-out using photodiodes and amplified and counted using custom-designed electronics. The detector system meet the requirements of high efficiency, good spatial resolution and low cost.

\section{Acknowledgments}

The authors gratefully acknowledge the financial support of the Australian Customs Service. The authors wish to thank the staff of the On-Line Analysis and Control Program of CSIRO Minerals for their invaluable contribution to the project.

\section{References}

[1] J.E. Eberhardt et al, Fast Neutron and Gamma-Ray Interrogation of Air Cargo Containers, these proceedings

[2] J.E. Eberhardt et al. Fast neutron radiography scanner for the detection of contraband in air cargo containers, Applied Radiation and Isotopes 63 (2005) 179

[3] J.I.W. Watterson, R.M. Ambrosi and H. Rahmanian, The detector problem in fast neutron radiography, $16^{\text {th }}$ Internat. Conf. on Applications of Accelerators in Research and Industry, AIP CP576, 2001, 1087.

[4] V.I. Mikerov et al, Prospects for efficient detectors for fast neutron imaging, Applied Radiation and Isotopes 61 (2004) 529

[5] R.C. Lanza, New trends in neutron radiography, in Application of Accelerators in Research and Industry, AIP Conference Proceedings 680, eds. J.L. Duggan and I.L. Morgan, August 2003, ISBN 0-7354-0149-7

[6] V. Dangendorf et al, Detectors for energy resolved fast neutron imaging, Nuclear Instruments and Methods in Physics Research A 535 (2004) 93

[7] J.E.Eberhardt, R.D.Ryan and A.J.Tavendale, Evaluation of epitaxial $n$-Ga-As for nuclear radiation detectors, Nuclear Instruments and Methods 94 (1971) 463.

[8] F. Tonetto et al, Optimizing performances of $\mathrm{CsI}(\mathrm{Tl})$ crystals with a photodiode readout, Nuclear Instruments and Methods in Physics Research A 420 (1999) 181

[9] J. Brose, G. Dahlinger and K.R. Schubert, Properties of CSI(Tl) crystals and their optimization for calorimetry of high energy photons, Nuclear Instruments and Methods in Physics Research A 417 (1998) 311 

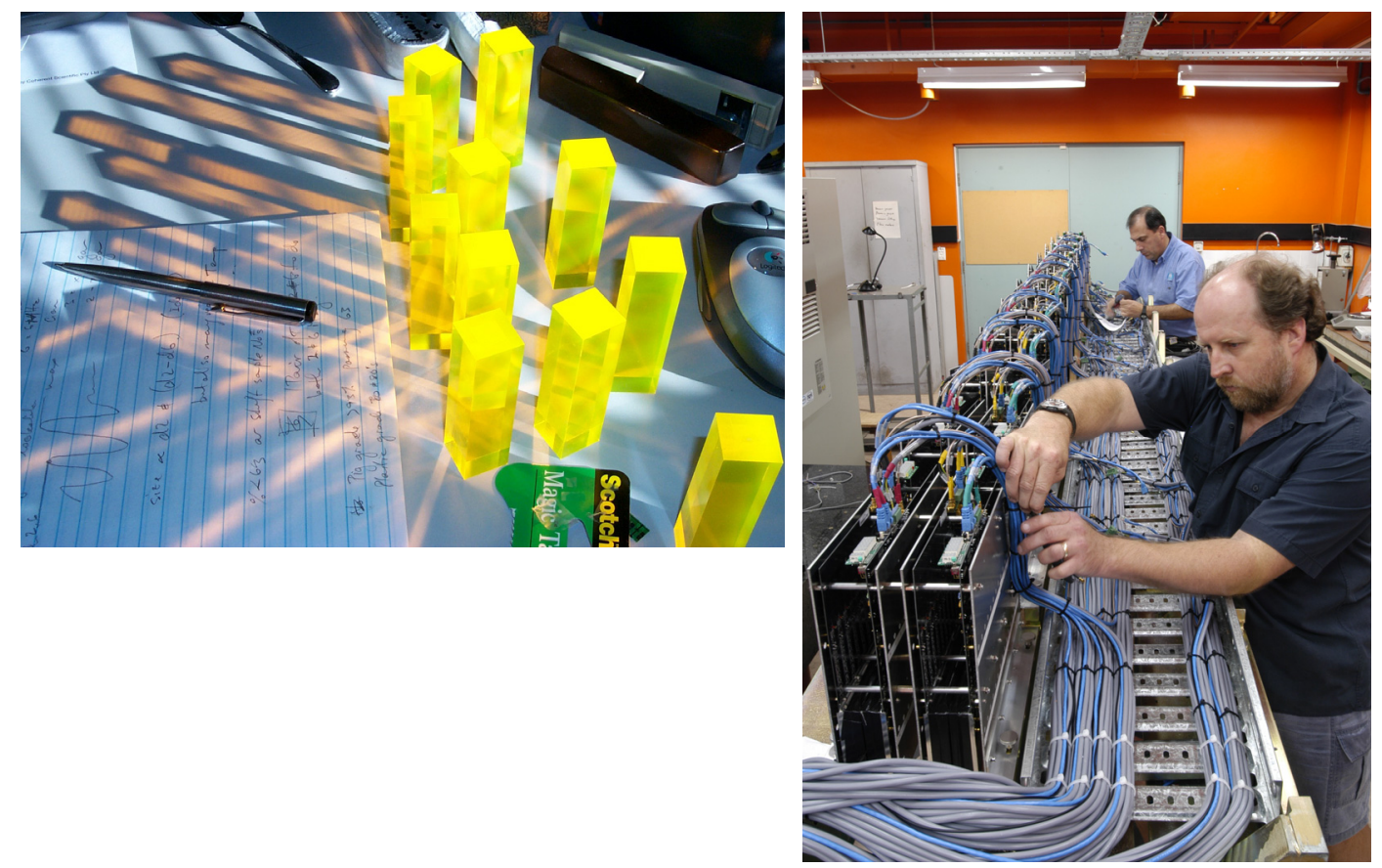

Figure 1. Photograph of orange scintillators used in the neutron detector array in the CSIRO Air Cargo

Figure 2. Photograph of neutron detector boards Scanner ar Brisbane Airport being assembled for installation in the CSIRO Air Cargo Scanner ar Brisbane Airport

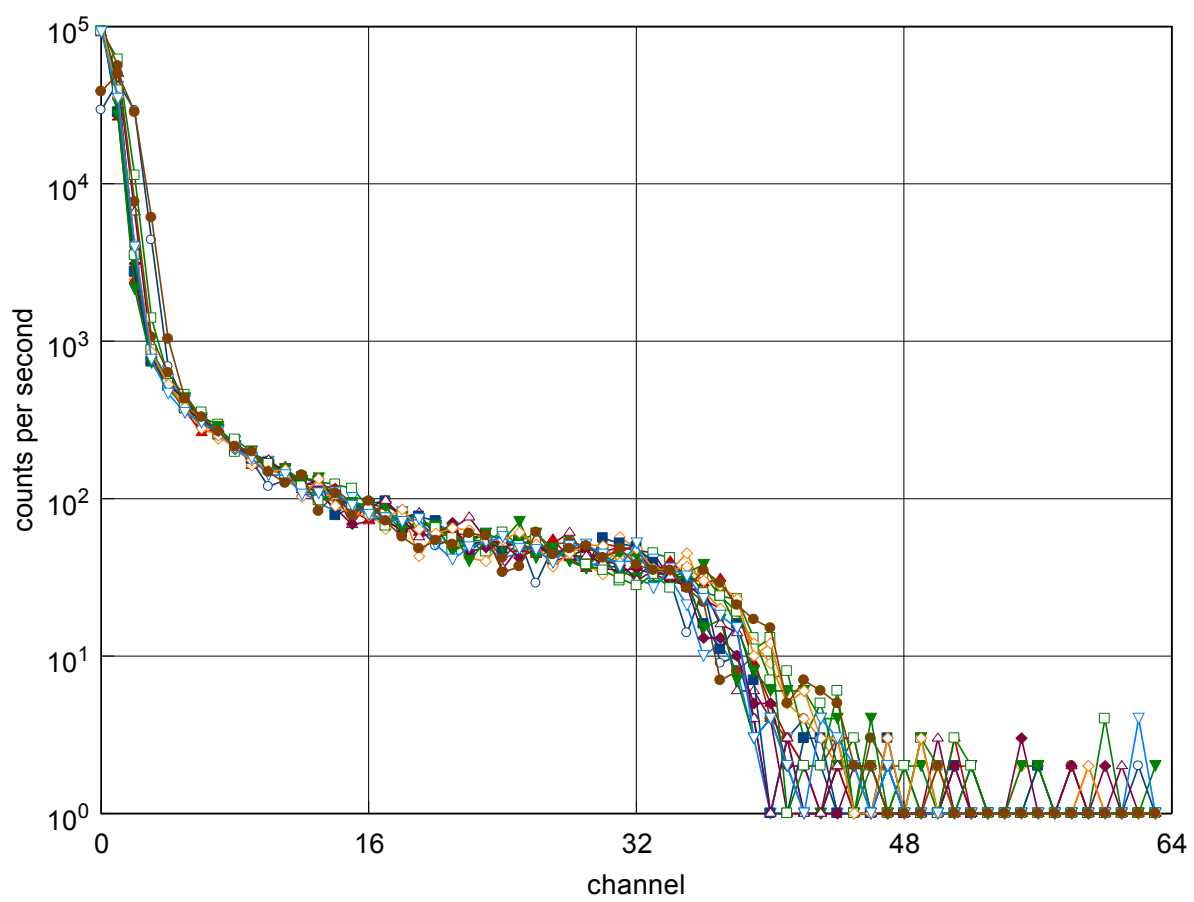

Figure 3. Pulse height spectra from all neutron detectors in one block of 16 detectors in the Brisbane scanner. 